\title{
Simultaneous EUV and radio observations of bidirectional plasmoids ejection during magnetic reconnection ${ }^{\star}$
}

\author{
Pankaj Kumar and Kyung-Suk Cho
}

\begin{abstract}
Korea Astronomy and Space Science Institute (KASI), 305-348 Daejeon, Republic of Korea e-mail: pankaj@kasi.re.kr
\end{abstract}

Received 24 December 2012 / Accepted 9 July 2013

\begin{abstract}
We present a multiwavelength study of the X-class flare, which occurred in active region (AR) NOAA 11339 on 3 November 2011. The extreme ultraviolet (EUV) images recorded by SDO/AIA show the activation of a remote filament (located north of the AR) with footpoint brightenings about $50 \mathrm{~min}$ prior to the flare's occurrence. The kinked filament rises up slowly, and after reaching a projected height of $\sim 49 \mathrm{Mm}$, it bends and falls freely near the AR, where the X-class flare was triggered. Dynamic radio spectrum from the Green Bank Solar Radio Burst Spectrometer (GBSRBS) shows simultaneous detection of both positive and negative drifting pulsating structures (DPSs) in the decimetric radio frequencies (500-1200 MHz) during the impulsive phase of the flare. The global negative DPSs in solar flares are generally interpreted as a signature of electron acceleration related to the upward-moving plasmoids in the solar corona. The EUV images from AIA 94 A reveal the ejection of multiple plasmoids, which move simultaneously upward and downward in the corona during the magnetic reconnection. The estimated speeds of the upward- and downward-moving plasmoids are $\sim 152-362$ and $\sim 83-254 \mathrm{~km} \mathrm{~s}^{-1}$, respectively. These observations strongly support the recent numerical simulations of the formation and interaction of multiple plasmoids due to tearing of the current-sheet structure. On the basis of our analysis, we suggest that the simultaneous detection of both the negative and positive DPSs is most likely generated by the interaction or coalescence of the multiple plasmoids moving upward and downward along the current-sheet structure during the magnetic reconnection process. Moreover, the differential emission measure (DEM) analysis of the active region reveals a hot flux-rope structure (visible in AIA 131 and $94 \AA$ ) prior to the flare initiation and ejection of the multitemperature plasmoids during the flare impulsive phase.
\end{abstract}

Key words. Sun: flares - Sun: radio radiation - Sun: magnetic topology

\section{Introduction}

It is well known that magnetic reconnection plays a key role in the release of the magnetic energy stored in sheared or twisted magnetic fields, leading to the onset of solar flares and coronal mass ejections (CMEs; Priest \& Forbes 2000; Aschwanden 2004). In the magnetic reconnection process, oppositely directed field lines break up and join again resulting in the release of magnetic energy into thermal energy and particle acceleration (e.g., Sweet 1958; Parker 1963; Petschek 1964). The standard model of a solar flare, known as the CSHKP model, explains the energy-release process (Carmichael 1964; Sturrock 1966; Hirayama 1974; Kopp \& Pneuman 1976), which is supported by several observational findings, such as cusp-shaped loops (Tsuneta et al. 1992), inflows and outflows (Yokoyama et al. 2001; Savage et al. 2010, 2012; Takasao et al. 2012), downflow signatures (McKenzie 2000; McKenzie \& Hudson 2001; Innes et al. 2003; Asai et al. 2004; Savage \& McKenzie 2011), plasmoid ejections (Shibata et al. 1995), loop-top hard X-ray sources (Masuda et al. 1994; Sui \& Holman 2003), X-ray jets (Shibata et al. 1992; Shimojo et al. 1996), bidirectional-jets (Innes et al. 1997), and flux rope or loop interactions (Kumar et al. 2010a,b,c; Török et al. 2011). According to the CSHKP model, the magnetic reconnection takes place in a vertical current sheet located above an underlying closed loop system, and the filament or prominence eruption plays a key role in the triggering of fast

\footnotetext{
* Movie is available in electronic form at http://www . aanda.org
}

reconnection (Forbes \& Lin 2000; Lin \& Forbes 2000; Kumar et al. 2012).

Although there are some fundamental problems in the theory of magnetic reconnection, for example, in the Sweet-Parker reconnection model (Sweet 1958; Parker 1957), the reconnectionrate is too slow $\left(10^{-4}-10^{-6}\right)$ to explain the magnetic energy release in solar flares. The Petschek (1964) reconnection model predicts a faster reconnection rate (i.e., 0.01-0.1), considering slow-mode MHD shocks in the outflow region. This model explains the observed reconnection rate in most of the solar flares. However, the observational value of the reconnection rate varies from 0.2-0.001 (Isobe et al. 2005; Narukage \& Shibata 2006; Takasao et al. 2012). MHD simulations have revealed that the localized resistivity can assist in obtaining the fast reconnection rate (i.e., close to unity) (Ugai \& Tsuda 1977; Yokoyama \& Shibata 1994). When the diffusion region becomes long enough (similar to the Sweet-Parker model), the tearing-mode instability can cause the onset of bursty reconnection, which involves the formation of several magnetic islands (i.e., plasmoids) in the current sheet (Furth et al. 1963; Kliem 1995; Priest \& Forbes 2000; Shibata \& Magara 2011).

Shibata et al. (1995) and Shibata \& Tanuma (2001) extended the CSHKP model by unifying reconnection and plasmoid ejection, and emphasized the importance of plasmoid ejection in the reconnection process, which is known as the "plasmoidinduced-reconnection" model. In this model, the bursty reconnection is triggered by a plasmoid ejection that leads to buildup of the magnetic energy in a vertical current sheet. When a 
plasmoid is ejected, the inflow is induced owing to the conservation of mass, which results in the enhancement of the reconnection rate. A plasmoid formed above the current sheet can also be accelerated by the faster reconnection outflow. Nishida et al. (2009) performed MHD simulations of the solar flares using different values of resistivity and plasmoid velocity, and find that the reconnection rate correlates positively with the plasmoid velocity. Therefore, the plasmoid ejection plays a key role in triggering fast magnetic reconnection and is observational evidence of magnetic reconnection in a solar flare. For example, in the soft X-ray and EUV images, upward-moving hot plasma blobs are frequently observed (Shibata et al. 1995; Ohyama \& Shibata 1998; Kim et al. 2005; Kumar et al. 2013; Kumar \& Manoharan 2013; Liu 2013), and the white-light coronagraph observations often show rising blob-like features in the wake of CMEs (Ko et al. 2003; Lin et al. 2005; Liu et al. 2010, 2011). The typical size of the plasmoid varies from $\sim 10^{9} \mathrm{~cm}$ (in compact flares) to $\sim 10^{11} \mathrm{~cm}$ (in CMEs), and their speed ranges between $\sim 10-1000 \mathrm{~km} \mathrm{~s}^{-1}$ (Shibata \& Tanuma 2001). Moreover, the size and velocity of multiple plasmoids formed during the the bursty reconnection (due to tearing instability) varies from $\sim 3-4^{\prime \prime}$ and $\sim 89-460 \mathrm{~km} \mathrm{~s}^{-1}$, respectively (Shen et al. 2011; Takasao et al. 2012). On the basis of the numerical simulation by Samtaney et al. (2009), the number of plasmoids (and spatial scales) formed from the tearing-mode instability depends on the Lundquist number (i.e., $\mathrm{S}^{\frac{3}{8}}$ ).

Drifting-pulsating structures (DPSs) are the slowly drifting radio spectrogram features of short durations $(\sim 1-3 \mathrm{~min})$, which consists of several quasi-periodic pulsations. They are generally observed in the decimetric frequency range, (e.g., $\sim 0.6-2.0 \mathrm{GHz}$ ) (Khan et al. 2002). Their mean bandwidth is about $500 \mathrm{MHz}$, and the frequency drift ranges from -20 to $-5 \mathrm{MHz} \mathrm{s}^{-1}$ (Karlický \& Bárta 2012). Based on a $2 \mathrm{D}$ numerical MHD simulation, Kliem et al. (2000) proposed that the DPSs are generated during a bursty magnetic-reconnection when interacting plasmoids are formed. They proposed that the electrons are accelerated during these processes and trapped in the plasmoids that generate individual pulses of the observed DPSs. Karlický (2004) used the concept of fractal reconnection proposed by Shibata \& Tanuma (2001) and considered several plasmoids to explain simultaneous observation of several DPSs. The interaction of plasmoids is also revealed in the numerical simulations, and negative or positive DPSs correspond to upwardor downward-moving plasmoids, respectively, thereby exciting the radio emissions within progressively lower or higher density regions (Bárta et al. 2007, 2008; Karlický \& Bárta 2007; Ning et al. 2007). According to Bárta et al. (2008), the upward and downward motions of the plasmoid is determined by the magnetic reconnection rate above and below the plasmoid. If the reconnection rate above the plasmoid is higher or lower than below, then the plasmoid moves downward or upward, respectively. The interaction of downward-moving plasmoids with the flare loop arcade or loop-top kernel are also noticed in the simulation and observation (Bárta et al. 2007; Riley et al. 2007; Kołomański \& Karlický 2007). Milligan et al. (2010) observed downward-moving plasmoid $\left(12 \mathrm{~km} \mathrm{~s}^{-1}\right)$ interacting with flare loop-top and showed the enhanced nonthermal emission in the corona at the time of the merging, suggesting the additional particle acceleration. Therefore, high resolution observations are extremely important for supporting the above simulation results.

So far, there has been no clear simultaneous observation of both the positive and negative DPSs associated with the multiple plasmoid ejections in EUV or X-ray images. Thanks to the SDO/AIA high-resolution observations, which made it possible to observe this rare phenomena for the first time in an X-class flare that occurred on 3 November 2011. In this paper, we present the simultaneous EUV and radio observational evidence of the bidirectional plasmoid ejections along the current-sheet structure during magnetic reconnection. In Sect. 2, we present the multiwavelength observational data analysis, and in the last section, we discuss our results.

\section{Observations and results}

The Atmospheric Imaging Assembly (AIA) instrument onboard the Solar Dynamics Observatory (SDO) mission observes full disk images of the Sun at a spatial resolution of $1.2^{\prime \prime}$, and its field of view covers $\sim 1.3 R_{\odot}$. In this study, we used AIA images observed in $171 \AA$ (Fe IX, $T \approx 0.7 \mathrm{MK}), 131 \AA$ (Fe VIII/XXI, $T \approx 0.4$ and $11 \mathrm{MK}), 94 \AA$ (Fe XVIII, $T \approx 7 \mathrm{MK}$ ), and $304 \AA$ (He II, $T \approx 0.05 \mathrm{MK})$. The AIA images observed at a $12 \mathrm{~s}$ cadence cover chromospheric to coronal heights (Lemen et al. 2012), which are useful for studying the evolutionary aspects of the X-class flare. We also used the Helioseismic and Magnetic Imager (HMI) magnetogram to study the magnetic field configuration at the flare site (Schou et al. 2012; Scherrer et al. 2012).

The top panel of Fig. 1 shows the GOES soft X-ray flux profile (1-8 $\AA$ channel) and the distance-time profiles of the upward and downward moving plasmoids (shown in Figs. 4 and 5). The middle panel displays the soft X-ray flux derivative profile. The bottom panel shows the $1 \mathrm{~s}$ cadence, relative radio flux density (in sfu) profiles at different frequencies $(1415,4995,8800$, and $15400 \mathrm{MHz}$ ) observed by the Sagamore Hill radio station (Straka 1977). The two stages of energy release is evident during the flare. The first-stage energy release (impulsive) was observed during 20:20-20:23 UT. Most of the nonthermal particles are accelerated during the first-stage energy release that peaks around 20:21 UT. The $1445 \mathrm{MHz}$ radio flux profile shows quasiperiodic oscillatory behavior. In addition, the radio emission at 4995,8800 , and $15400 \mathrm{MHz}$ is usually related to the gyrosynchrotron emission during the flare. It is likely that the radio emission mechanism at high frequencies $(>2 \mathrm{GHz})$ is considered to be caused by gyrosynchrotron emission, whereas plasma emission is below about $2 \mathrm{GHz}$ (Dulk 1985). The gradual second energy-release took place from 20:23 to 20:28 UT. The timing of the DPSs is indicated by two vertical dotted lines. It is important to note that the DPSs are observed during the first energy release and are separated well from the gyrosynchotron emission produced by the accelerated electrons during the magnetic reconnection.

\subsection{Filament activation}

AR NOAA 11339 was located at N18E49 on 3 November 2011 with the $\beta \gamma$ magnetic configuration. This active region produced seven $\mathrm{C}$-class, one M-class and one X-class flare on the same day. According to the Solar Geophysical Data (SGD) report, the X1.9 flare was started at 20:16 UT, peaked at 20:27 UT and ended at 20:32 UT. Figure 2 displays the selected snapshots of AIA $304 \AA$ channel, which represent the lower solar atmosphere, i.e., the chromosphere and transition region. These images show the preflare activities in and around the active region, where the $\mathrm{X}$-class flare was triggered. About $50 \mathrm{~min}$ prior to the flare, we noticed the activation of a remote-filament (at 19:30:08 UT) associated with small-scale brightening below it. The filament was located $\sim 50^{\prime \prime}$ away (toward the north) from the AR (indicated by the arrow). The activated filament slowly rises up and bends 
Pankaj Kumar and Kyung-Suk Cho: Bidirectional plasmoids ejection during magnetic reconnection

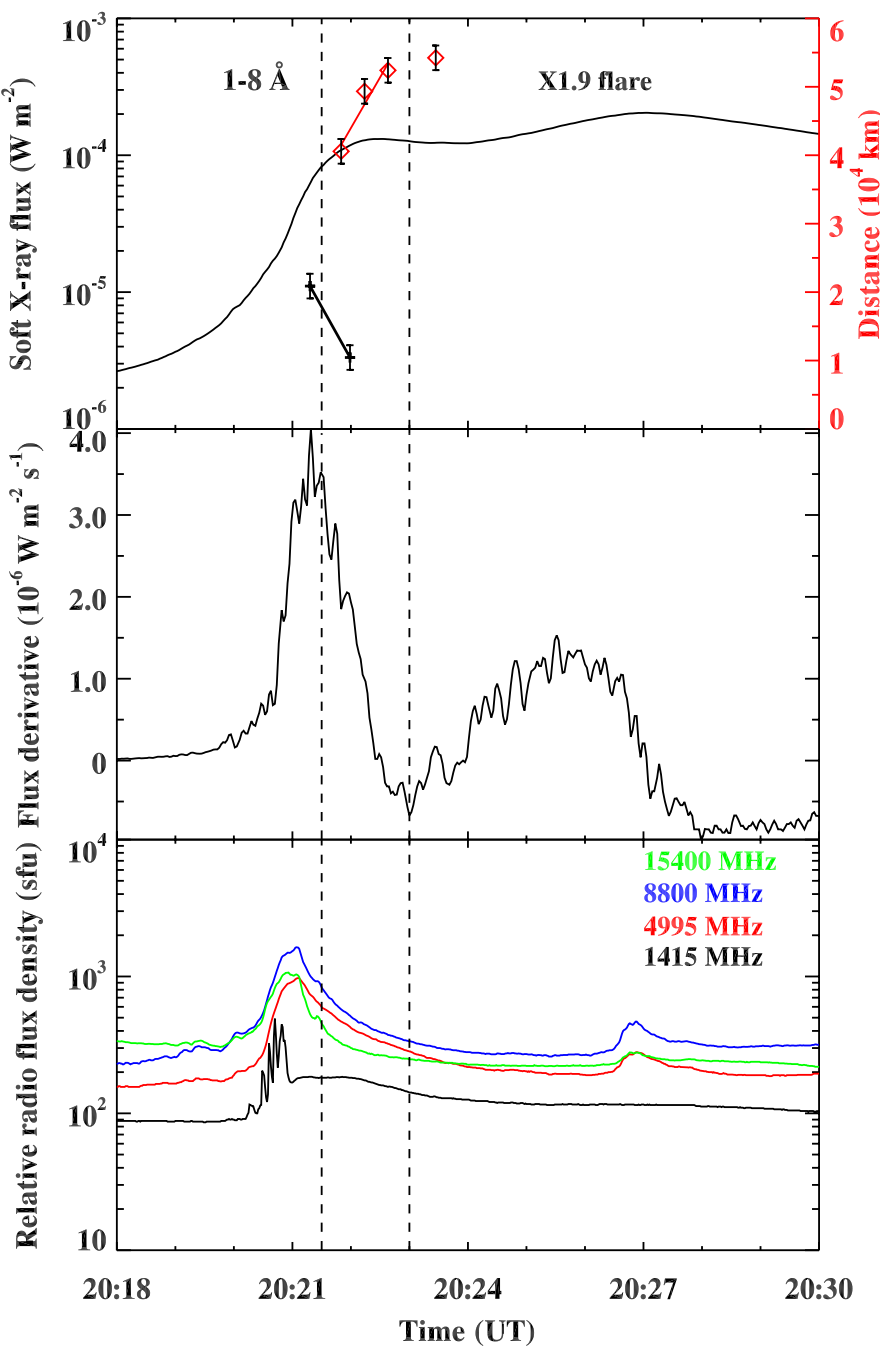

Fig. 1. Top: GOES soft X-ray flux in the $1-8 \AA$ channel and distancetime measurements of the upward- and downward- moving plasmoids. Middle: GOES soft X-ray flux derivative. Bottom: RSTN 1 s cadence radio flux profiles in $1445,4995,8800$, and $15400 \mathrm{MHz}$ frequencies during the flare on 3 November 2011. The two vertical dotted lines indicate the timings of the observed DPSs.

toward the AR after reaching a projected height of $\sim 49 \mathrm{Mm}$ (see image at 19:35:08 UT). The AIA $304 \AA$ intensity movie clearly shows the plasma flows along the filament channel from the activation site (direction is shown by arrows). At 19:50:08 UT, we observed plasma flows along "A" and "B" directions toward the AR site. The filament system started fading after 20:00 UT, but the plasma flows continued (marked by arrow). The flare was initiated at 20:16 UT, where the plasma flows along the filament channel were dissipated. The flare ribbons were observed at 20:20:08 UT (shown by the arrows). To investigate the photospheric magnetic field configuration at the flare site, we overlaid the HMI magnetogram image contours of positive (white) and negative (black) polarities at the top of AIA $304 \AA$ image at 20:10:08 UT. This image suggests that the filament activation and brightening took place at the location of the weak magnetic flux, where no sunspot was observed in the HMI.

To explore the coronal magnetic configuration of the AR, we used AIA $131 \AA$ images, which are shown in the bottom panel of Fig. 2. The AIA $131 \AA$ channel is sensitive to both cool $(\sim 0.4 \mathrm{MK})$ and hot $(\sim 11 \mathrm{MK})$ plasma. The first panel at 19:30:33 UT shows the activation and rising of a remote kinked (indicated by "K") filament. Apparently the kinked filament shows one turn. AIA $131 \AA$ movie reveals the presence of a flux-rope structure (above the flare site) prior to the flare initiation. The flux rope was only visible in the AIA hot channels (i.e., 131 and $94 \AA$ ), which is marked by "hot FR". An underlying hot loop is evident at the flare site. The footpoints of the underlying hot loop are indicated by arrows, where the flare ribbons were formed. In the second panel at 19:50:45 UT, we observe the downward motion of the filament apex ("F") near the hot flux-rope (FR) structure, where the flare was triggered. In addition, we also notice the downward motion of the plasma along "B". We observed an underlying hot flare loop at 20:18:33 UT, nearby where the downward moving plasma along the filament channel disappeared. During the early phase of the $\mathrm{X}$-class flare, we noticed the upward motion of the higher loops (see AIA $131 \AA$ intensity movie, Fig. 2) and the downward motion of the underlying hot loop. The downward plasma flows along the activated filament channel are likely to have some effect or interaction (i.e., field destabilization) with the ambient magnetic field configuration at the flare site, although the present observations are not sufficient to predict the exact triggering mechanism of the flare.

To examine the temporal and spatial evolution of the plasma flows towards the AR, we used a slice cut "S1" (AIA $131 \AA$ image shown in the bottom panel of Fig. 2) along the bending filament channel. The space-time intensity plot along with the GOES soft X-ray flux profile (in 1-8 $\AA$ wavelength channel) is displayed in Fig. 3. The stack plot reveals the plasma flows along the filament channel until 20:14 UT (indicated by the arrows). We observed two peaks in the flux profile at $\sim 20: 22$ UT and $\sim 20: 27$ UT, which reveal the two stages of energy release during the flare. The second peak is more intense, which represents the $\mathrm{X}$-class flare. The plasmoid ejections (observed in EUV) took place during the first impulsive energy release.

\subsection{Plasmoid ejections}

Figure 4 displays the selected snapshots of the AIA 171, 131, and $94 \AA$ during the flare impulsive phase. The first panel (at 20:21:50 UT) shows the ejection of a plasmoid (marked by an arrow) from the flare site, which continues to rise. Later, we observe multiple plasmoid ejections during the flare impulsive phase (shown in the AIA $171 \AA$ panel at 20:23:27 UT). AIA $171 \AA$ intensity movie shows the interaction and coalescence of multiple plasmoids with each other. The typical thickness of the plasmoids is $\sim 3-4^{\prime \prime}$. In the bottom panels, we observe a bright underlying flare loop and higher loops in the AIA 131 and $94 \AA$ at 20:18:33 UT. At 20:22:37 UT, multiple plasmoid ejections are observed above the hot underlying flare loop. A vertical structure above the underlying hot loop may be the current sheet (in AIA 131 and $94 \AA$ ) and the plasmoids are formed and ejected along the sheet structure. The plasmoids were observed in both hot and cool AIA channels, which suggests the multithermal nature of the plasmoids. The plasmoids were impulsively ejected from the current sheet, and finally diffused into the corona following a deceleration trend. Some of the plasmoids move downward in the corona after reaching a certain height.

To estimate the rising speed of the plasmoid, we tracked the topmost plasmoid (from the flare center) in the AIA $171 \AA$ images shown in the top panels. The distance-time profile of this plasmoid is shown in Fig. 1. Using the linear fit to the data points, the mean speed of the plasmoid was about $247 \mathrm{~km} \mathrm{~s}^{-1}$. 

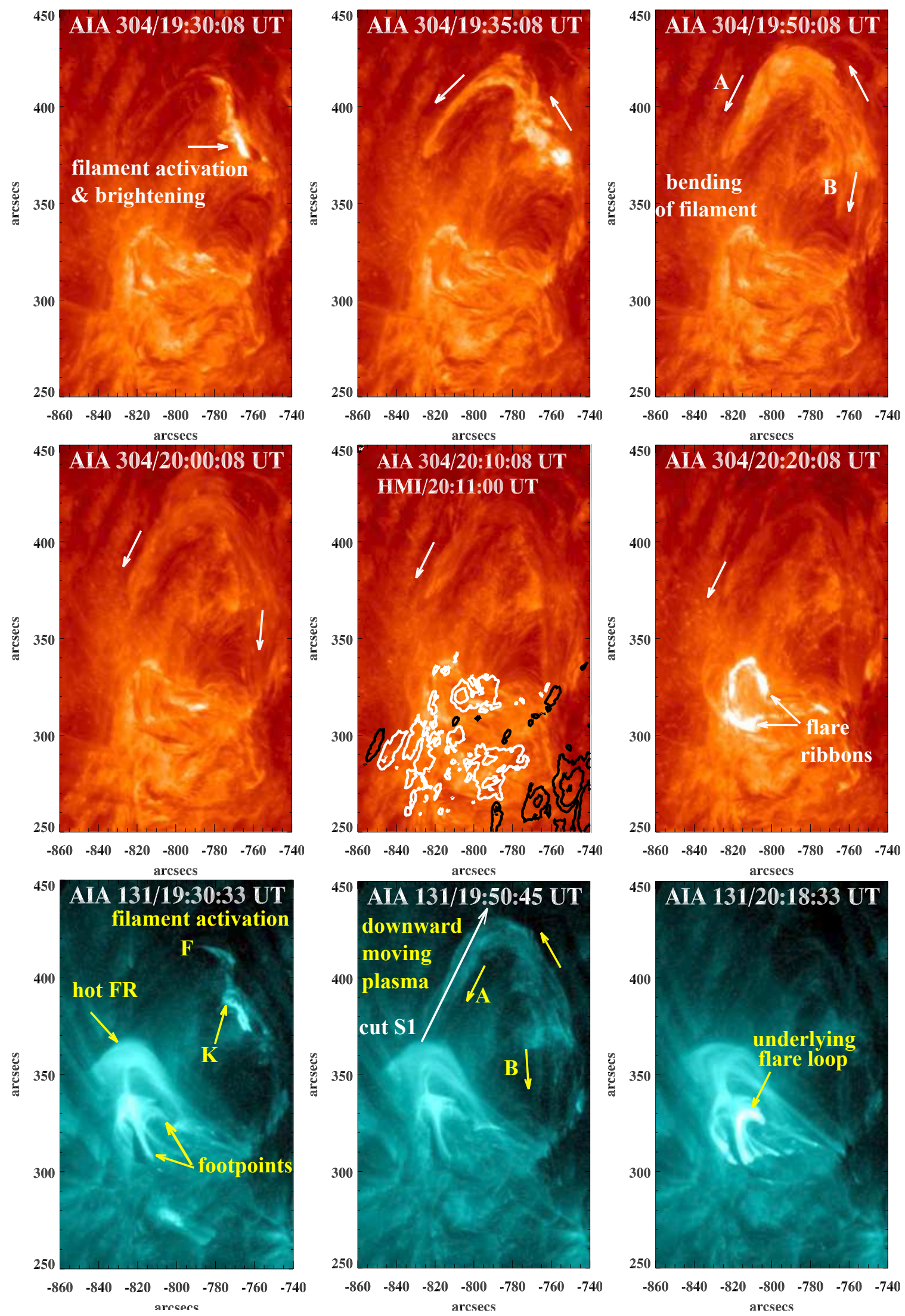

Fig. 2. SDO/AIA EUV images at $304 \AA$ (top and middle) and $131 \AA$ (bottom) showing activation and bending of the filament toward the AR hot flux-rope structure on 3 November 2011. AIA $304 \AA$ image at 20:10:08 UT is overlaid by the HMI magnetogram contours of positive (white) and negative (black) polarities. The contour levels are $\pm 500, \pm 1000$, and $\pm 2000 \mathrm{G}$. The temporal evolution in the $304 \AA$ and $131 \AA$ bands over about one hour until the flare sets in can be found in a movie available in the online edition. 


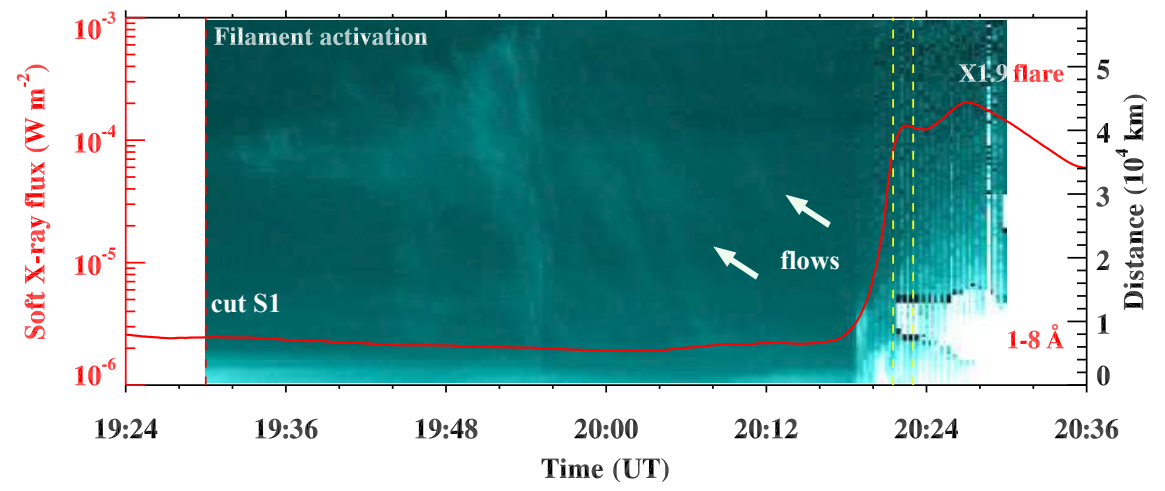

Fig. 3. GOES soft X-ray flux profile in the 1-8 Å channel and space-time plot along slice "S1" of the AIA $131 \AA$ images on 3 November 2011. A vertical red dotted line at $\sim 19: 30 \mathrm{UT}$, indicates the timing of filament activation and associated brightening. Two vertical dashed lines (yellow) indicate the timing of multiple plasmoid ejections during the impulsive phase of the flare. The timing of DPSs and plasmoid ejection is consistent with each other (see Fig. 1).
We assume a four-pixel (2.4") error in the distance measurement for the visually tracked topmost plasmoid. The estimated error in the mean speed is $\sim 74 \mathrm{~km} \mathrm{~s}^{-1}$. It should be noted that the measured speed is biased by the projection effect and is the lower limit of the true speed of the plasmoid in 3D.

\subsection{Inflows/outflows}

We used slice cuts "S2" and "S3" on the AIA $131 \AA$ image to investigate the inflow and outflow structures related to the flare. The directions for slices are chosen after many trials to observe the clear signature of inflow and outflow patterns. The left hand panel of Fig. 5 shows the space-time intensity distribution plots for slices "S2" and "S3". During the initial phase of the flare, we observed the apparent motion $\left(\sim 55 \mathrm{~km} \mathrm{~s}^{-1}\right)$ of the eastern loop toward the current-sheet structure (above the underlying hot loop). At the same time, we observed the upward motion $\left(\sim 56 \mathrm{~km} \mathrm{~s}^{-1}\right)$ of the higher loops and downward motion $\left(\sim 46 \mathrm{~km} \mathrm{~s}^{-1}\right)$ of the underlying hot-loop system (slice "S3"). These apparent motions of the loop systems may be indirectly linked to the inflow and outflow related to the magnetic reconnection. The outflow speed is quite low during the initial phase of the flare, which is probably the apparent motion of the hotloop systems. In a well cited event, Yokoyama et al. (2001) found the inflow velocities of $1.0-4.7 \mathrm{~km} \mathrm{~s}^{-1}$ by tracking the motion of patterns above the limb using SOHO/EIT images. Furthermore, Narukage \& Shibata (2006) reported inflow speeds ranging from $2.6-38 \mathrm{~km} \mathrm{~s}^{-1}$ in a statistical study of six flare events. Recently, using the SDO/AIA observations, Savage et al. (2012) found the inflow speeds the order of $100 \mathrm{~km} \mathrm{~s}^{-1}$ and the outflow speeds from $\sim 100$ to $1000 \mathrm{~km} \mathrm{~s}^{-1}$. In our observations, the speed of the pattern (i.e., inflow speed) is higher than reported by Yokoyama et al. (2001), but lower than by Savage et al. (2012). However, the event studied by Savage et al. (2012) was located on the eastern limb, which may account for the higher inflow velocities since the projection effects are minimal.

We observed the shrinkage of the underlying flare loop $\left(\sim 46 \mathrm{~km} \mathrm{~s}^{-1}\right)$ about two minutes prior to the radio emission peaks. Simultaneously, we also observed the outward motion of the higher loop $\left(\sim 56 \mathrm{~km} \mathrm{~s}^{-1}\right)$ and the apparent inflow motion of the loop $\left(\sim 55 \mathrm{~km} \mathrm{~s}^{-1}\right)$. It seems that the current sheet is formed in between the underlying flare loop and the higher loop system. Futhermore, the motion of the loops (upwards or downwards) reveal the signature of magnetic reconnection during the early phase of the flare. Sui \& Holman (2003) reported the loop shrinkage (speed $\sim 9 \mathrm{~km} \mathrm{~s}^{-1}$, for 2-4 $\mathrm{min}$ ) before the hard X-ray peak, which was most likely caused by the change in the magnetic field configuration as the X-point collapsed into the current sheet. Similarly, Li \& Gan (2005) found the flare loop shrinkage ( $34 \mathrm{GHz}$ radio images, speed $13 \mathrm{~km} \mathrm{~s}^{-1}$ ) in the rising phase of about nine minutes. Our observational results are consistent with the above reported events, but the shrinkage speed is higher in our case. However, we do not have the radio and hard X-ray imaging observations to study the source motion in more detail.

Some of the AIA images in other wavelengths (except $94 \AA$ ) showed saturation at the flare center during the impulsive phase of the flare. Therefore, it was not possible to observe the downward-moving plasmoids in these wavelengths. To observe the simultaneous upward and downward motion of the plasmoids during the flare impulsive phase, we created the stack plots along slices S4 and S5 using AIA $94 \AA$ images (marked in the bottom right panel of Fig. 4). The start and end points for these slices (in arcsecs) are taken as (i) S4 $=(-803,315),(-850$, $390)$; (ii) S5 $=(-804,300)$ and $(-836,400)$.

The right hand panel of Fig. 5 displays the stack plots along slices S4 (top) and S5 (bottom) during 20:18-20:30 UT. Interestingly, we observed the upward- and downward-moving plasmoids simultaneously. We estimated the speed of the upward and downward-moving plasmoid along slice S5. The initial speed of the upward-moving plasmoid is $\sim 362 \mathrm{~km} \mathrm{~s}^{-1}$, whereas the speed of the downward moving plasmoid is $\sim 254 \mathrm{~km} \mathrm{~s}^{-1}$. These speeds are the initial speeds of the plasmoid, which are tracked in the space-time plot. The speed of the upward moving plasmoid is slightly more than the average speed $\left(\sim 247 \mathrm{~km} \mathrm{~s}^{-1}\right)$ of the topmost plasmoid as reported in the previous section. The calculated speed of the other plasmoids (along slice S4) is $\sim 152 \mathrm{~km} \mathrm{~s}^{-1}$ (upward) and $\sim 83 \mathrm{~km} \mathrm{~s}^{-1}$ (downward).

The characteristics of the flare (i.e., inflow and outflow) are consistent with the standard flare model (i.e., CSHKP). To determine the reconnection rate from our observations, we consider the outflow speed equal to the Alfvén speed, i.e., $V_{\mathrm{o}}=V_{\mathrm{A}}$ as predicted in the two-dimensional magnetic reconnection theories (Priest \& Forbes 2000), assuming that the speed of pattern $\left(\sim 55 \mathrm{~km} \mathrm{~s}^{-1}\right)$ roughly equivalent to the inflow speed. The reconnection rate is $M_{\mathrm{A}}=\frac{V_{\mathrm{i}}}{V_{\mathrm{o}}} \sim 0.22$, for the outflow speed of $\sim 247 \mathrm{~km} \mathrm{~s}^{-1}$, which is consistent with the reconnection rate reported in Takasao et al. (2012). This reveals the fast reconnection as predicted in Petschek (1964) reconnection model. Several plasma blobs appeared in the current-sheet structure, then collided with one another and were ejected from it. On the basis of the observational findings, we consider that the sheet structure is the current sheet, and the plasma blobs are the magnetic islands/plasmoids created by the tearing mode instability as suggested by Takasao et al. (2012).

\subsection{Drifting pulsating structures (DPSs)}

Figure 6 shows the dynamic radio spectrum obtained from the Green Bank Solar Radio Burst Spectrometer (GBSRBS) ranging 

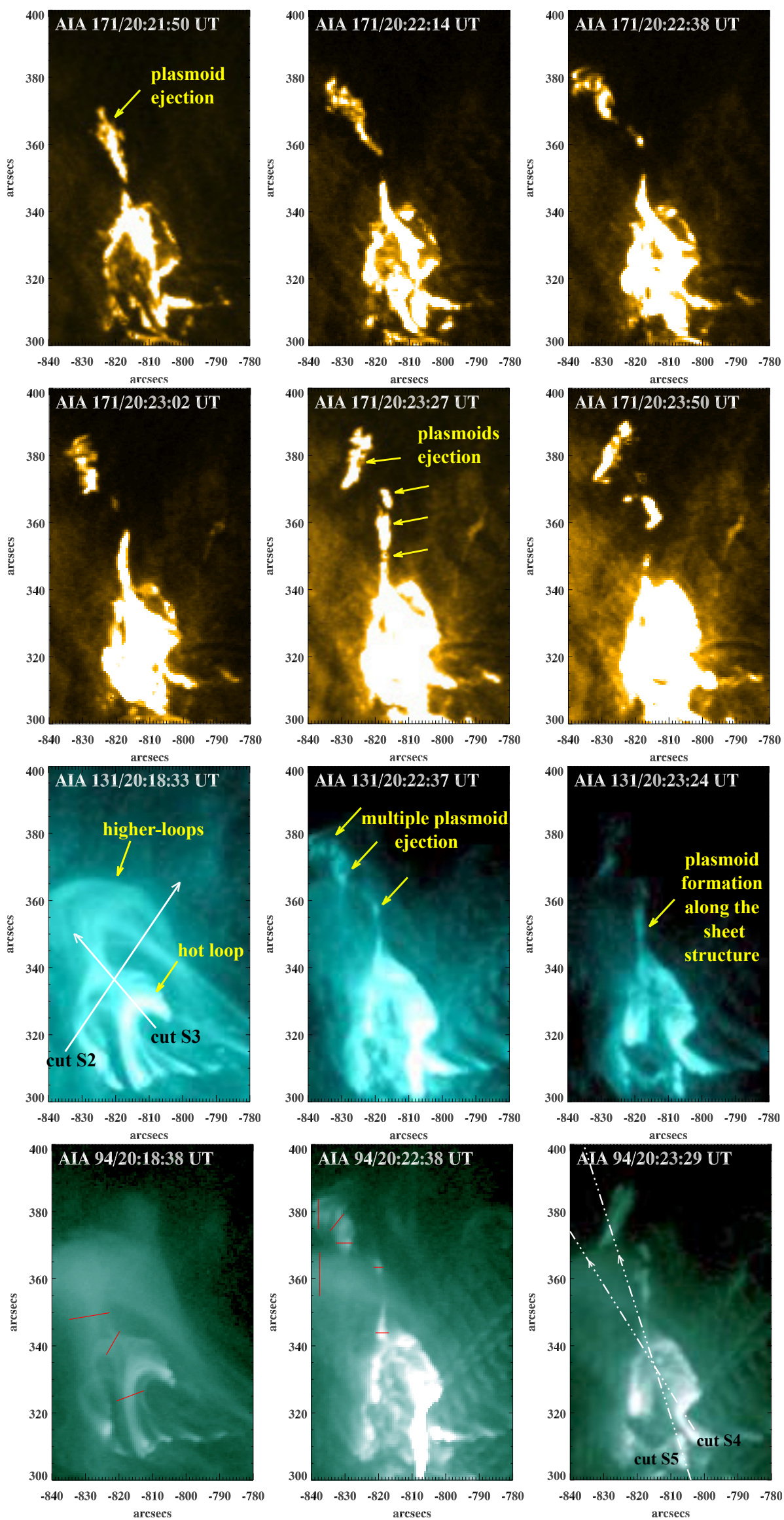

Fig. 4. SDO/AIA EUV images at 171 (top two rows), 131 (middle), and 94 (bottom) $\AA$ showing multiple plasmoid ejections during the X-class flare on 3 November 2011. Red lines in the AIA $94 \AA$ images (bottom) indicate the approximate thickness of the structures that is used for the density estimation. 
Pankaj Kumar and Kyung-Suk Cho: Bidirectional plasmoids ejection during magnetic reconnection
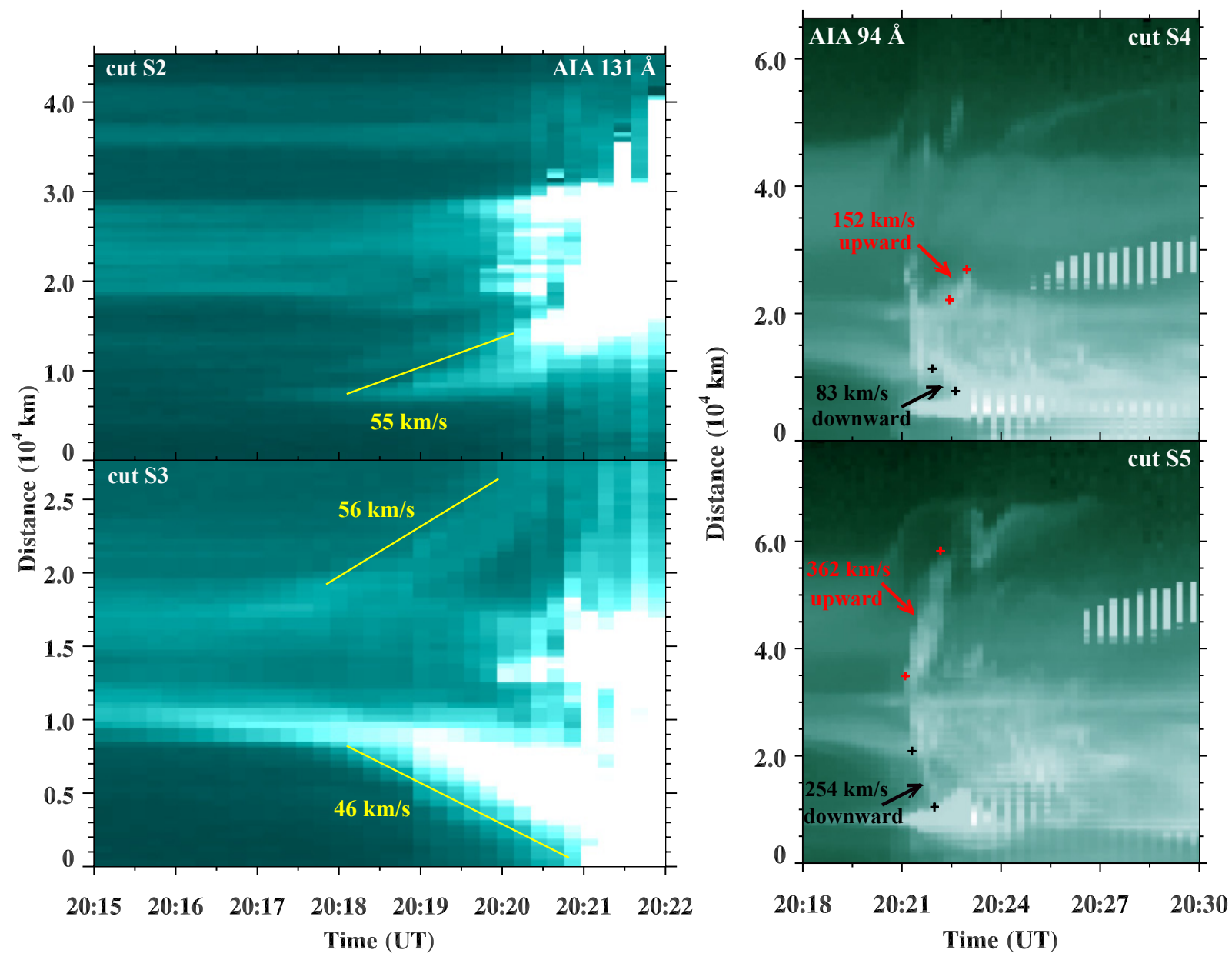

Fig. 5. Space-time intensity plots along slices "S2", "S3", "S4", and "S5" for the AIA 131 (left) and 94 (right) A images, shown in Fig. 4. The positions of the upward- and downward-moving plasmoid is marked by red and black "+" symbols.

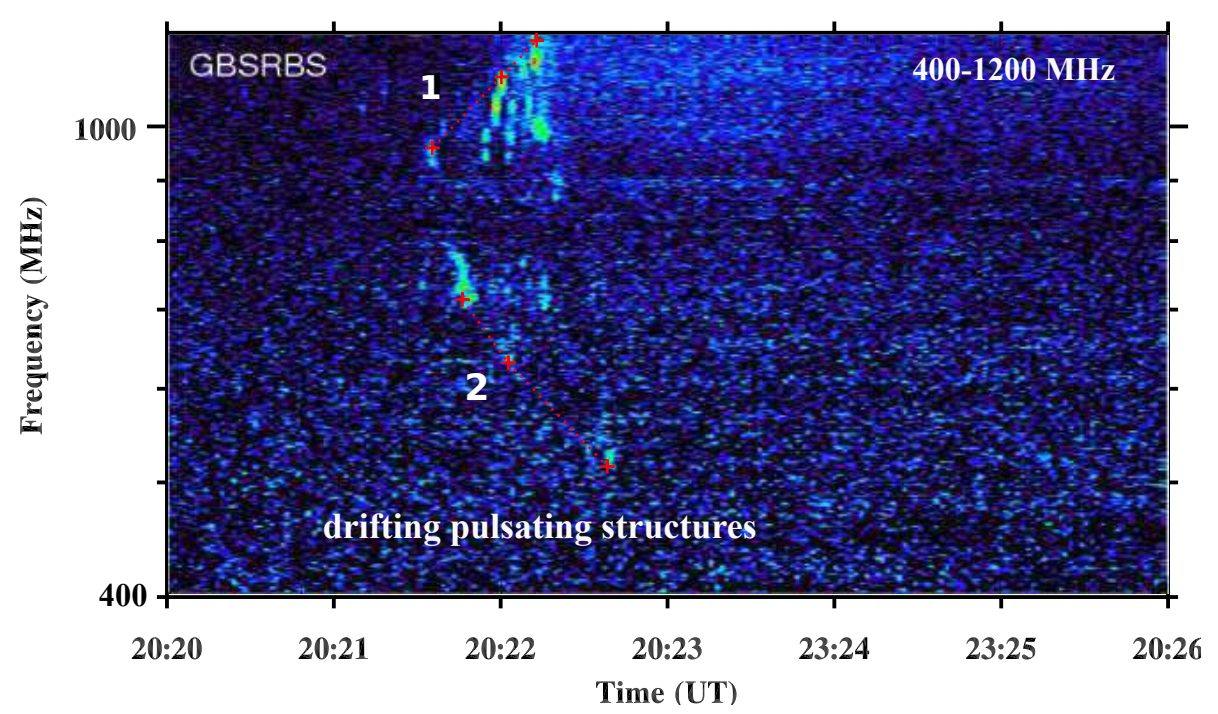

Fig. 6. Dynamic radio spectrum from the GBSRBS showing both the positive (marked by "1") and negative ("2") drifting pulsating structures (DPSs) during the X-class flare on 3 November 2011. from 400 to $1200 \mathrm{MHz}$ (decimetric) with a $1 \mathrm{~s}$ time resolution. The spectrometer is located in a radio-quiet zone at NRAO's Green Bank site, therefore it produces a highly sensitive dynamic spectra at low-noise radio interference. We observed drifting pulsating structures (DPSs) during the flare impulsive phase
(20:21:24 UT-20:22:36 UT) for about 1 min duration. The positive and negative DPSs are marked by " 1 " and " 2 ", respectively. It is interesting to note that at the same time, we observed multiple plasmoid ejections in the AIA images (see Fig. 4). Therefore, the DPSs are closely related with the dynamics of 
multiple plasmoid ejections. The negative DPSs are generally observed during the upward moving plasmoids and can be used to derive the source speed.

The plasma frequency is related with the local electron density by $f=9 \sqrt{ } n_{\mathrm{e}} \mathrm{MHz}$, where $n_{\mathrm{e}}$ is the electron density in $\mathrm{m}^{-3}$. The frequency drift rate is defined as

$$
\frac{\mathrm{d} f}{\mathrm{~d} t} \simeq \frac{f}{2 H} v
$$

where $H=\left|n_{\mathrm{e}} / \frac{\mathrm{d} n_{\mathrm{e}}}{\mathrm{d} r}\right|$ is the inhomogeneous plasma density scale height in the source and $v=\frac{\mathrm{d} r}{\mathrm{~d} t}$ is the speed of the moving emission source (Pohjolainen et al. 2007; Wang et al. 2012). Then, the source speed can be estimated by using

$v=2 \frac{1}{f} \frac{\mathrm{d} f}{\mathrm{~d} t} H$

where $\frac{1}{f} \frac{\mathrm{d} f}{\mathrm{~d} t}$ is the relative frequency drift rate $\left(\mathrm{s}^{-1}\right)$. To estimate the drift rates for the DPSs, we selected few data points at the emission lane (marked by "+" symbol) in the dynamic spectrum and fitted a straight line to get the slopes, i.e., frequency drift rates. The estimated relative frequency drift rates are 0.0055 and $-0.0061 \mathrm{~s}^{-1}$, respectively for " 1 " and " 2 ". In a different DPSs event, Khan et al. (2002) measured the frequency drift rate of $-2.8 \mathrm{MHz} \mathrm{s}^{-1}$ at a mean frequency of $430 \mathrm{MHz}$. Our calculated relative negative drift rate is comparable to the value of about $-0.0065 \mathrm{~s}^{-1}$ in Khan et al. (2002). The positive drift represents the downward moving emission source. From the AIA observations, the speed of upward-moving plasmoid is $\sim 247 \mathrm{~km} \mathrm{~s}^{-1}$ (Figs. 4 and 1). Therefore, using Eq. (2), we obtain the scale height $H=2.0 \times 10^{4} \mathrm{~km}$. This value agrees with the density scale heights reported by Aschwanden \& Benz (1986) $\left(H \sim(2-20) \times 10^{3} \mathrm{~km}\right)$ by analyzing several type III radio bursts and pulsations in a frequency range of 100-1000 MHz. Using the above scale height, we obtained the speed of the downward moving radio source $\sim 224 \mathrm{~km} \mathrm{~s}^{-1}$. These are the mean speed of the upward- and downward-moving radio sources for the observed DPSs. The speed of the downward-moving radio source (from radio observation) is less than the speed of the upward moving source, i.e., plasmoid. The observed drifting-pulsating emissions are likely to be generated by the plasma emission. The positive and negative DPSs imply the plasma densities of $1.13 \times 10^{10}$ to $1.72 \times 10^{10} \mathrm{~cm}^{-3}$ and $3.2 \times 10^{9}$ to $6.2 \times 10^{9} \mathrm{~cm}^{-3}$, respectively.

To determine the speed of the DPS exciter independently from the coronal density model, we use the Newkirk one-fold and two-fold density models (Newkirk 1961). But, these models do not provide the reliable heights of the emission source, which lies below the heliocentric distance of $1 R_{\odot}$. It means that the emission source is located below the solar surface, which is not correct. Alternatively, we use a barometric isothermal density law for the local density scale height, which is generally used for the decimetric emission (Pohjolainen et al. 2007, 2008). In this method, the local electron density $\left(n_{\mathrm{e}}\right)$ is given by

$$
n_{\mathrm{e}}=N_{\mathrm{e}} \exp \left(-\frac{696000}{H}\left(1-\frac{1}{R}\right)\right)
$$

where $N_{\mathrm{e}}$ is the reference electron density at the base of the corona, and $\mathrm{R}$ the estimated heliocentric distance (Demoulin $\&$ Klein 2000). If we assume the reference electron density of $10^{9} \mathrm{~cm}^{-3}$, a local scale height of $2 \times 10^{4} \mathrm{~km}$, and the local electron densities from the DPS, the estimated exciter speed for the positive and negative DPS are 194 and $228 \mathrm{~km} \mathrm{~s}^{-1}$, respectively. However, the speeds are closer to these estimated speeds, but the source heights are still below the heliocentric distance of $1 R_{\odot}$ $\left(0.92-0.93 R_{\odot}\right)$. Therefore, we would like to mention that without proper normalization, density models may not provide the true height of the exciter in the case of the high frequency decimetric bursts (i.e., type II) or drifting pulses (Bain et al. 2012; Cho et al. 2013).

\subsection{Differential emission measure (DEM) analysis of the active region}

To determine the peak temperature and emission measure of the active region, we utilized AIA images in six coronal filters (i.e., $94,171,131,211,335$, and $193 \AA$ ). We used the SSWIDL code developed by Aschwanden et al. (2011) for this purpose. The coalignment of AIA images at six coronal EUV wavelengths is carried out by using the limb fitting method, with an accuracy of $<1$ pixel. The code fits a DEM solution in each pixel (or macro-pixel), which can be parametrized by a single Gaussian function that has three free parameters: the peak emission measure $\left(\mathrm{EM}_{\mathrm{p}}\right)$, the peak temperature $\left(T_{\mathrm{p}}\right)$, and the temperature width sigma $\left(\sigma_{\mathrm{T}}\right)$. The peak emission measure $\left(\mathrm{cm}^{-5} \mathrm{~K}^{-1}\right)$ and temperature $(\mathrm{MK})$ maps (during the flare and plasmoids eruption) are shown in Fig. 7. Furthermore, to estimate the density of the hot coronal loop, the current-sheet structure, and the multiple plasmoids, we selected small boxes that are shown in the top and bottom panels of Fig. 7. First of all, we calculated the average temperature, EM and $\sigma_{T}$ in the selected regions. Using these values, we estimated the total emission measure (TEM) in the selected regions using the formula $\int D E M(T) \mathrm{d} T$. Using the values of total EM $\left(\mathrm{cm}^{-5}\right)$, we estimated the densities of the selected structures in the active region, assuming that the depth of the structure along the line of sight is nearly equal to its width (Cheng et al. 2012). If " $L$ " is the width of the structure, then the density $\left(n_{\mathrm{e}}\right)$ of the structure is calculated by using the relation $n_{\mathrm{e}}=\sqrt{\frac{E M}{L}}$ (assuming filling factor $\left.\approx 1\right)$. The width of structures is marked in the AIA $94 \AA$ images (Fig. 4, bottom panels). All the estimated values are summarized and listed in Table 1.

In Fig. 7, we displayed the peak emission measure and the peak temperature maps at 20:18:38 UT (top) and 20:22:38 UT (bottom). The top panels show a dense, hot underlying loop, a possible current-sheet structure, and a higher loop system above it. The estimated mean temperature within the selected regions (indicated by $1,2,3$ ) are $5.37,6.30$, and $8.31 \mathrm{MK}$, respectively. This reveals quite a high temperature for these loop structures, which are observed only in AIA 131 and $94 \AA$ channels. This indicates the presence of a hot flux rope before triggering of the flare. The computed mean density within these regions are $\sim 9.73 \times 10^{9}, \sim 1.35 \times 10^{10}$, and $\sim 1.63 \times 10^{10} \mathrm{~cm}^{-3}$, respectively. The bottom panels illustrate the ejection of multiple plasmoids along the current-sheet structure. The ejection of hot plasma possibly from the current-sheet structure is evident, which is marked by " 1 ". The peak temperature and density of the ejected hot plasma are $\sim 7.2 \mathrm{MK}$ and $\sim 8.65 \times 10^{9} \mathrm{~cm}^{-3}$, respectively. The rest boxes from " 2 " to " 6 " show the multiple plasmoid ejections along the current sheet. It is interesting to note that the cool and hot plasma blobs are detected along the current-sheet structure. The peak temperature of the plasma blobs varies from $\sim 1.6$ to $3.4 \mathrm{MK}$, whereas the density varies from $\sim 8.54 \times 10^{9}$ to $\sim 2.76 \times 10^{10} \mathrm{~cm}^{-3}$. Although we could observe the downward-moving plasmoids in the AIA $94 \AA$ images, it was not possible to track these plasmoids on the temperature maps due to the image saturation in other AIA channels. 
Pankaj Kumar and Kyung-Suk Cho: Bidirectional plasmoids ejection during magnetic reconnection

Table 1. Temperature (MK) and density $\left(\mathrm{cm}^{-3}\right)$ distributions within the selected regions.

\begin{tabular}{lccccccc}
\hline \hline Date/time(UT) & boxes & $\log \left(T_{\mathrm{p}}\right)(\mathrm{MK})$ & $\log \left(E M_{\mathrm{p}}\right)\left(\mathrm{cm}^{-5} \mathrm{~K}^{-1}\right)$ & $\sigma_{\mathrm{T}}$ & $\mathrm{TEM}\left(\mathrm{cm}^{-5}\right)$ & $L$ & $n_{\mathrm{e}}\left(\mathrm{cm}^{-3}\right)$ \\
\hline $03 / 11 / 201120: 18: 38$ & 1 & 6.73 & 22.28 & 0.149 & $8.63 \times 10^{28}$ & $12^{\prime \prime}$ & $9.73 \times 10^{9}$ \\
& 2 & 6.80 & 22.31 & 0.149 & $1.10 \times 10^{29}$ & $8^{\prime \prime}$ & $1.35 \times 10^{10}$ \\
& 3 & 6.92 & 22.39 & 0.149 & $1.71 \times 10^{29}$ & $8.5^{\prime \prime}$ & $1.63 \times 10^{10}$ \\
\hline $03 / 11 / 201120: 22: 38$ & 1 & 6.86 & 22.01 & 0.170 & $7.30 \times 10^{28}$ & $13^{\prime \prime}$ & $8.65 \times 10^{9}$ \\
& 2 & 6.29 & 22.41 & 0.170 & $4.92 \times 10^{28}$ & $9^{\prime \prime}$ & $8.54 \times 10^{9}$ \\
& 3 & 6.54 & 22.10 & 0.170 & $4.24 \times 10^{28}$ & $6.4^{\prime \prime}$ & $9.39 \times 10^{9}$ \\
& 4 & 6.22 & 22.58 & 0.170 & $6.19 \times 10^{28}$ & $5^{\prime \prime}$ & $1.28 \times 10^{10}$ \\
& 5 & 6.24 & 22.42 & 0.170 & $4.45 \times 10^{28}$ & $3^{\prime \prime}$ & $1.40 \times 10^{10}$ \\
& 6 & 6.28 & 23.09 & 0.170 & $2.28 \times 10^{29}$ & $4^{\prime \prime}$ & $2.76 \times 10^{10}$ \\
\hline 18/08/2010 05:10:50 & 1 & 6.39 & 21.90 & 0.175 & $2.01 \times 10^{28}$ & $2^{\prime \prime}$ & $1.15 \times 10^{10}$ \\
& 2 & 6.30 & 22.13 & 0.175 & $2.76 \times 10^{28}$ & $6^{\prime \prime}$ & $7.83 \times 10^{9}$ \\
& 3 & 6.18 & 22.03 & 0.175 & $1.62 \times 10^{28}$ & $3^{\prime \prime}$ & $8.5 \times 10^{9}$ \\
\hline
\end{tabular}
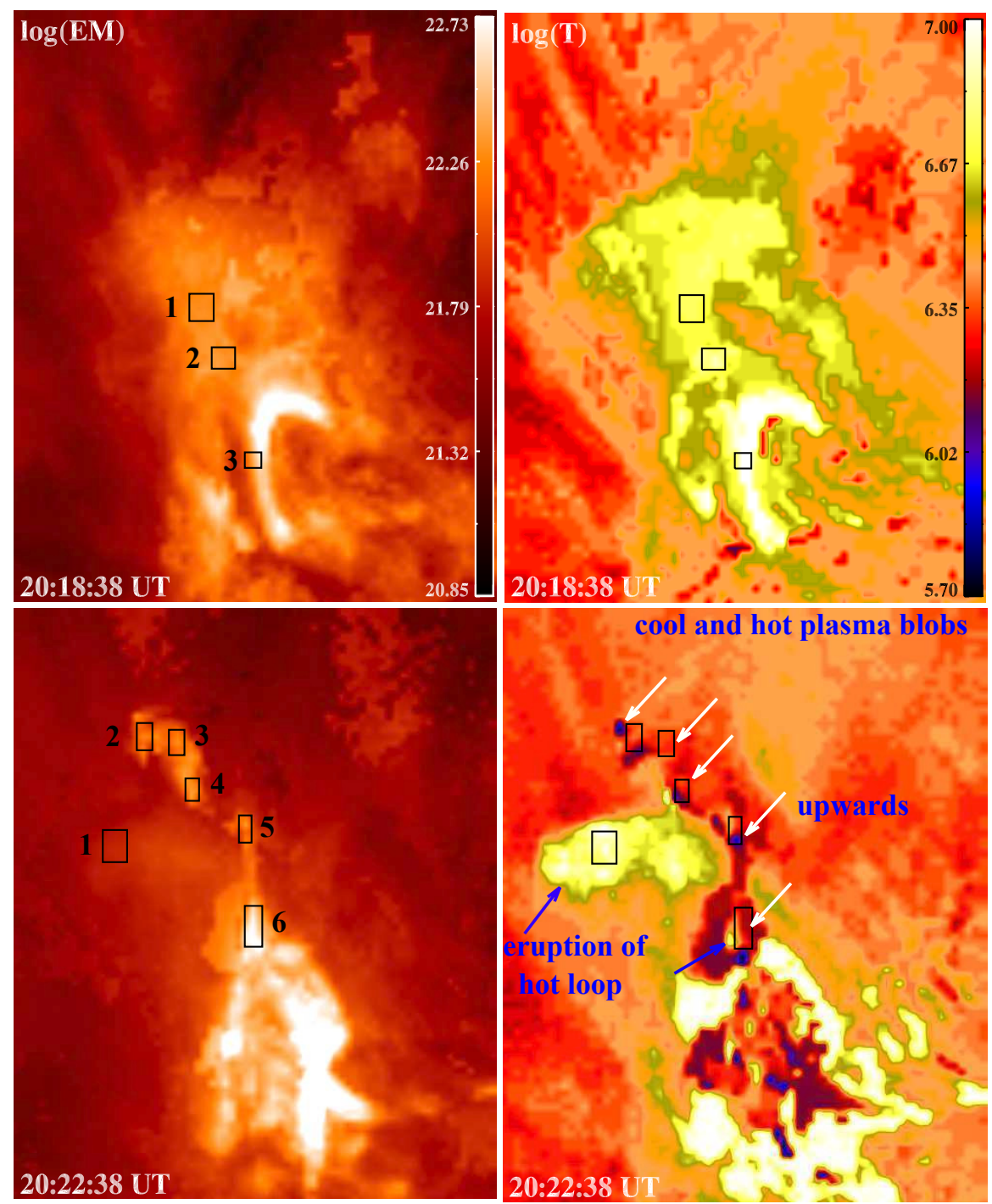

Fig. 7. Peak emission measure $\left(\mathrm{cm}^{-5} \mathrm{~K}^{-1}\right)$ and temperature (MK) maps for the active region during the flare on 3 November 2011. The size of each image is $85^{\prime \prime} \times 104^{\prime \prime}$.
A similar type of bidirectional multiple plasmoid ejections associated with inflows has recently been reported by Takasao et al. (2012) in a C4.5 limb flare occurred in AR NOAA 11099 on 18 August 2010. The plasma blobs were observed both in hot and cool AIA channels during the impulsive phase of the flare similar to our event. The top panels of Fig. 8 (i.e., AIA 131 and $94 \AA$ images) show the underlying hot loop and the ejection of multiple plasmoids above it, along the possible current-sheet structure. To compare the characteristics of the plasma blobs in both events, we generate the peak temperature and emission maps for AR NOAA 11099 , which is shown in the bottom panel of Fig. 8. The peak temperature and density of the plasma blobs in regions 1,2 , and 3 varies from $\sim 1.5$ to $2.4 \mathrm{MK}$ and $\sim 7.83 \times 10^{9}$ to $1.15 \times 10^{10} \mathrm{~cm}^{-3}$, respectively. On 


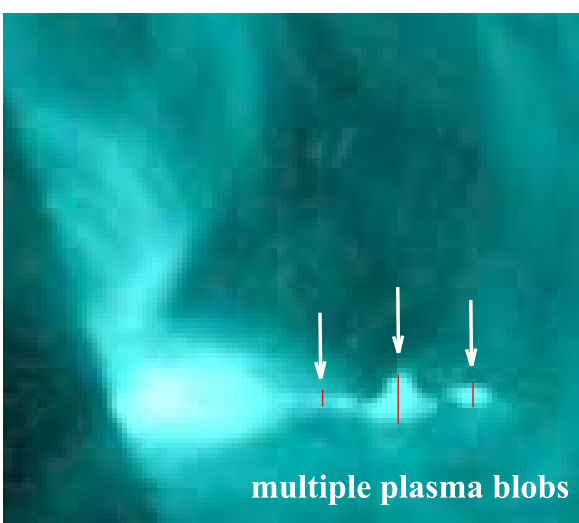

AIA 131/05:10:45 UT
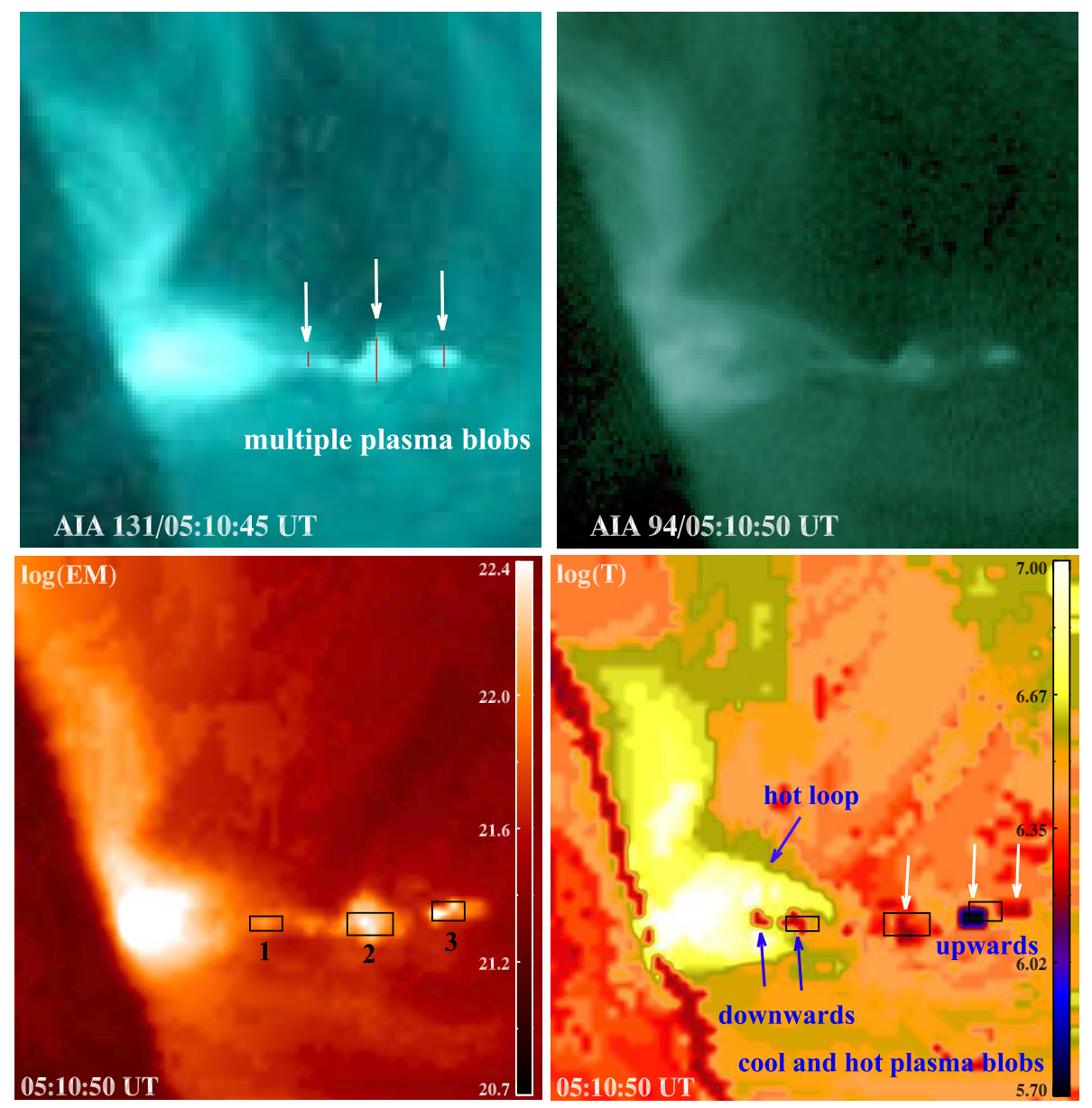

Fig. 8. Top: AIA 131 and $94 \AA$ images showing the ejection of multiple plasmoids. The red lines in the first panel indicate the approximate thickness of the plasmoids. Bottom: peak emission measure $\left(\mathrm{cm}^{-5} \mathrm{~K}^{-1}\right)$ and temperature (MK) maps for the active region during C4.5 flare occurred on 18 August 2010. The size of each image is $81^{\prime \prime} \times 84^{\prime \prime}$.
18 August 2010, a prominence eruption also took place near the flare site, similar to our event. Later, we observed the flare and ejection of multiple plasmoids. The generation of tearing instability and the ejection of multiple plasmoids are quite similar in both of the events. Although the radio observations for the 18 August 2010 event are not available, it would have been useful to compare the dynamic radio spectrum for both events.

Interestingly, we observe the multitemperature plasmoids $(\sim 1.6-3.4 \mathrm{MK})$ formed by the tearing of the current sheet during the magnetic reconnection. The observed temperature of multiple X-ray plasmoids (in a different event) associated with the individual peak of hard X-ray emission was $\sim 10 \mathrm{MK}$ (Nishizuka et al. 2010). The estimated temperature and density of a X-ray plasmoid observed in a 5 October 1992 flare was $\sim 6-13 \mathrm{MK}$ and $\sim 8-15 \times 10^{9} \mathrm{~cm}^{-3}$, respectively (Ohyama $\&$ Shibata 1998). In our case, the observed temperature of the plasmoids is lower than that of reported in the previous studies. However, the density of the plasmoids is comparable. In addition, the temperature of the plasmoid was lower than that of the region in between the plasmoid and the underlying flare loop, which is consistent with the magnetic reconnection model (Yokoyama \& Shibata 1997, 1998). The formation of multitemperature plasmoids during the tearing mode instability is not clear (observed here for the first time) and further investigations are needed.

\section{Discussion and conclusion}

We studied multiwavelength observations of the X-class flare that occurred on 3 November 2011. The associated energy-release below an activated filament induced the plasma flows along the filament channel. Furthermore, the downwardmoving filament apex towards and close to the AR possibly destabilized the magnetic field configuration at the flare site leading to the flare onset. The plasma flows along the filament channel slow down about $15-20$ min prior to the flare occurrence. The downward-moving plasma flows are likely to be responsible for the onset of inflows needed for the flare initiation. As shown in the numerical simulation by Shen et al. (2011), an initial perturbation is applied to the current sheet to induce the inflow. The AIA images reveal an underlying hot loop before the flare impulsive phase and the formation of a current sheet possibly above the underlying hot loop. Generation of the tearing mode instability in the current sheet leads to the formation and coalescence of multiple plasmoids (Furth et al. 1963; Shibata \& Tanuma 2001). The radio observations confirm both positive and negative DPSs simultaneously during 20:21:24 UT-20:22:36 UT. This suggests the signature of electron acceleration associated with the multiple bidirectional plasmoid ejections (observed in the AIA images) from the reconnection site. The interaction and coalescence of a series of plasmoids leads to the effective particle acceleration and associated plasma heating (Karlický \& Bárta 2007).

Recently, the AIA observations revealed the formation of a hot flux-rope structure prior to and during the solar eruption (Cheng et al. 2011; Zhang et al. 2012), which was considered as a driver of the solar eruption. In the solar flare event observed on 3 November 2010, a hot vertical current sheet was formed below an erupting flux-rope structure (observed in AIA hot channels, i.e., 131 and $94 \AA$ A) (Reeves \& Golub 2011; Cheng et al. 2011; 


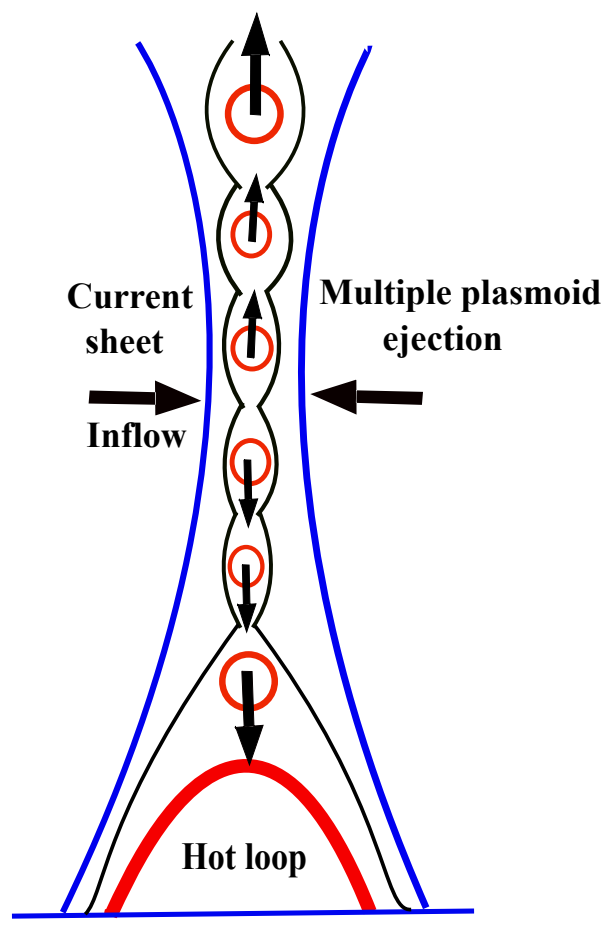

Fig. 9. Schematic cartoon showing the bidirectional plasmoid ejections along the current sheet structure during magnetic reconnection.

Kumar \& Innes 2013; Hannah \& Kontar 2013). Patsourakos et al. (2013) also reported the formation of a hot flux rope prior to an eruption, and its subsequent destabilization leads to the onset of a flare/CME. Therefore, in our event, hot structure above the underlying flare loop (observed in the 131 and $94 \AA$ ) is the flux rope structure, which is formed prior to the flare initiation. A current sheet is probably formed in between the hot flux-rope and underlying flare loop. As discussed by Aschwanden (2004), a resistive instability is generated in the current sheet, when the driving forces of the inflow exceed the opposing Lorentz force. These driving forces are produced by the sheared magnetic fields leading to the onset of tearing mode instability (Furth et al. 1963).

Shen et al. (2011) have performed the resistive MHD simulations to study the internal structure of the current sheets. In their simulation, reconnection rate becomes faster when the magnetic islands or plasma blobs are formed, and the plasmoids move in both directions (up and down) along the current sheet, whose speeds range from $147-242 \mathrm{~km} \mathrm{~s}^{-1}$ for upward and $89-159 \mathrm{~km} \mathrm{~s}^{-1}$ for downward- moving plasmoids. In our case, the mean speed of the upward- and downward-moving plasmoids are $\sim 247$ and $\sim 224 \mathrm{~km} \mathrm{~s}^{-1}$, respectively. The speed of the downward-moving plasmoids is less than the upwardmoving plsamoids. This may be because the closed flare loops prevent blobs from moving faster in the downward or sunward direction (Shen et al. 2011). Following the Shibata and Karlický models, we draw a schematic cartoon (Fig. 9) to explain the scenario of the event. An underlying flare loop, inflows, and the bidirectional multiple plasmoid ejections along the current sheet structure are shown.

Using the AIA observations of a limb flare, Takasao et al. (2012) observed the signature of inflows and outflows associated with bidirectional plasmoid ejections. The typical size of the plasmoid in their event was about $2-3^{\prime \prime}$, and the velocities of upward and downward ejection were $220-460 \mathrm{~km} \mathrm{~s}^{-1}$ and $250-280 \mathrm{~km} \mathrm{~s}^{-1}$, respectively. The shape, sizes, and speed of the plasmoids (thickness about 3-4") in our event are nearly consistent with their results. Takasao et al. (2012) could observe the downward-moving plasmoids due to a limb event (negligible projection effect). Similarly, we also observed the downward motion of the plasmoids in the AIA $94 \AA$ images, and the downward motion of the plasmoids is confirmed by the positive DPSs observed in the dynamic radio spectrum. Multiple plasmoids/magnetic islands are created probably by tearing-mode instability in the current sheet, which collided with each other and were ejected from it. The plasmoids were visible in AIA hot (131 and $94 \AA$ ) and cool (304 $\AA$ ) channels, suggesting their multithermal nature. Very likely the plasmoids were heated by the coalescence process (Kliem et al. 2000). The plasmoid ejections and DPSs are observed during the first impulsive energy release. These plasmoids can induce strong inflow along with the enhancement of the reconnection rate (Shibata \& Tanuma 2001). Therefore, the ejection of multiple plasmoids played an important role in triggering the second energy release.

Ning et al. (2007) also observed the positive DPSs during a flare on 18 March 2003 and suggested that these are probably caused by the downward moving plasmoid in the corona. Numerical simulation results also show that the downward motion of the plasmoid is possible in the current sheet during a bursty magnetic reconnection (Karlický \& Bárta 2007; Shen et al. 2011). Our observational finding of simultaneous upward- and downward-moving multiple plasmoids supports their interpretation. As discussed in the numerical simulation by Karlický et al. (2010), the pulses of electromagnetic emission are generated at a location between two interacting plasmoids just before the coalescence of two plasmoids into a larger one. They find that the Langmuir waves accumulate in the interacting plasmoids at the locations of superthermal electrons (Drake et al. 2005; Karlický \& Bárta 2007). On the other hand, the electromagnetic waves appear at the boundaries of the plasmoids and then move outward, where they mutually interfere and generate the short-period pulsations. Using the particle-incell (PIC) model with periodic boundary conditions, Oka et al. (2010) also found the efficient electron acceleration by a multiisland coalescence process. Therefore, we expect that the electron acceleration takes place in between the plasmoids during the coalescence/merging of the plasmoids as shown in the model of Karlický (2004). Moreover, densities of the plasmoid regions derived with the AIA images are consistent with the densities corresponding to the positive and negative DPSs. Thus, our observations agree with the suggestions and interpretations given by Karlický et al. (2010) and Karlický \& Bárta (2011).

However, in recent numerical simulations (Bárta et al. 2008; Shen et al. 2011), both the negative and positive DPSs have been suggested as associated with the upward- and downward-moving plasmoids, respectively, but the simultaneous oppositely directed DPSs in radio, associated with a series of plasmoids in EUV, have not been reported earlier.

In conclusion, we reported the simultaneous radio and EUV observations of the multiple plasmoid ejections that moved bidirectionally along the current sheet structure during magnetic reconnection. Furthermore, the high-resolution observations (from Hinode and SDO) of similar types of events, combined with radio observations, will be helpful for understanding the characteristics of these plasma blobs and associated particle acceleration in more detail.

Acknowledgements. We would like to thank the anonymous referee for constructive comments, suggestions and help with interpreting the data, which improved the manuscript considerably. SDO is a mission for NASA's Living With a 
Star (LWS) program. National Radio Astronomy Observatory (NRAO) is operated for the NSF by Associated Universities, Inc., under a cooperative agreement. P.K. thanks P.K. Manoharan and D.E. Innes for several helpful discussions. This work has been supported by the "Development of Korea Space Weather Center" project of KASI, and the KASI basic research fund.

\section{References}

Asai, A., Yokoyama, T., Shimojo, M., \& Shibata, K. 2004, ApJ, 605, L77

Aschwanden, M. J. 2004, Physics of the Solar Corona. An Introduction (Praxis Publishing Ltd)

Aschwanden, M. J., \& Benz, A. O. 1986, A\&A, 158, 102

Aschwanden, M. J., Boerner, P., Schrijver, C. J., \& Malanushenko, A. 2011, Sol. Phys., 384

Bain, H. M., Krucker, S., Glesener, L., \& Lin, R. P. 2012, ApJ, 750, 44

Bárta, M., Karlický, M., Vršnak, B., \& Goossens, M. 2007, Centr. Eur. Astrophys. Bull., 31, 165

Bárta, M., Vršnak, B., \& Karlický, M. 2008, A\&A, 477, 649

Carmichael, H. 1964, NASA Spec. Publ., 50, 451

Cheng, X., Zhang, J., Liu, Y., \& Ding, M. D. 2011, ApJ, 732, L25

Cheng, X., Zhang, J., Saar, S. H., \& Ding, M. D. 2012, ApJ, 761, 62

Cho, K.-S., Gopalswamy, N., Kwon, R.-Y., Kim, R.-S., \& Yashiro, S. 2013, ApJ, 765,148

Demoulin, P., \& Klein, K.-L. 2000, in Transport and Energy Conversion in the Heliosphere, eds. J. P. Rozelot, L. Klein, \& J.-C. Vial (Berlin: Springer Verlag), Lect. Notes Phys. 553, 99

Drake, J. F., Shay, M. A., Thongthai, W., \& Swisdak, M. 2005, Phys. Rev. Lett., 94, 095001

Dulk, G. A. 1985, ARA\&A, 23, 169

Forbes, T. G., \& Lin, J. 2000, J. Atmos. Sol.-Terr. Phys., 62, 1499

Furth, H. P., Killeen, J., \& Rosenbluth, M. N. 1963, Physics of Fluids, 6, 459

Hannah, I. G., \& Kontar, E. P. 2013, A\&A, 553, A10

Hirayama, T. 1974, Sol. Phys., 34, 323

Innes, D. E., Inhester, B., Axford, W. I., \& Wilhelm, K. 1997, Nature, 386, 811

Innes, D. E., McKenzie, D. E., \& Wang, T. 2003, Sol. Phys., 217, 267

Isobe, H., Takasaki, H., \& Shibata, K. 2005, ApJ, 632, 1184

Karlický, M. 2004, A\&A, 417, 325

Karlický, M., \& Bárta, M. 2007, A\&A, 464, 735

Karlický, M., \& Bárta, M. 2011, ApJ, 733, 107

Karlický, M., \& Bárta, M. 2012, in Hinode-3: The 3rd Hinode Science Meeting, ed. T. Sekii, T. Watanabe, \& T. Sakurai, ASP Conf. Ser., 454, 287

Karlický, M., Bárta, M., \& Rybák, J. 2010, A\&A, 514, A28

Khan, J. I., Vilmer, N., Saint-Hilaire, P., \& Benz, A. O. 2002, A\&A, 388, 363

Kim, Y.-H., Moon, Y.-J., Cho, K.-S., Bong, S.-C., \& Park, Y. D. 2005, ApJ, 635, 1291

Kliem, B. 1995, in Coronal Magnetic Energy Releases, eds. A. O. Benz, \& A. Krüger (Berlin: Springer Verlag), Lect. Notes Phys., 444, 93

Kliem, B., Karlický, M., \& Benz, A. O. 2000, A\&A, 360, 715

Ko, Y.-K., Raymond, J. C., Lin, J., et al. 2003, ApJ, 594, 1068

Kołomański, S., \& Karlický, M. 2007, A\&A, 475, 685

Kopp, R. A., \& Pneuman, G. W. 1976, Sol. Phys., 50, 85

Kumar, P., \& Innes, D. E. 2013, Sol. Phys.

Kumar, P., \& Manoharan, P. K. 2013, A\&A, 553, A109

Kumar, P., Manoharan, P. K., \& Uddin, W. 2010a, ApJ, 710, 1195

Kumar, P., Srivastava, A. K., Filippov, B., \& Uddin, W. 2010b, Sol. Phys., 266, 39

Kumar, P., Srivastava, A. K., Somov, B. V., et al. 2010c, ApJ, 723, 1651

Kumar, P., Cho, K.-S., Bong, S.-C., Park, S.-H., \& Kim, Y. H. 2012, ApJ, 746, 67

Kumar, P., Park, S.-H., Cho, K.-S., \& Bong, S.-C. 2013, Sol. Phys., 282, 503
Lemen, J. R., Title, A. M., Akin, D. J., et al. 2012, Sol. Phys., 275, 17

Li, Y. P., \& Gan, W. Q. 2005, ApJ, 629, L137

Lin, J., \& Forbes, T. G. 2000, J. Geophys. Res., 105, 2375

Lin, J., Ko, Y.-K., Sui, L., et al. 2005, ApJ, 622, 1251

Liu, R. 2013, MNRAS

Liu, R., Lee, J., Wang, T., et al. 2010, ApJ, 723, L28

Liu, R., Wang, T.-J., Lee, J., et al. 2011, RA\&A, 11, 1209

Masuda, S., Kosugi, T., Hara, H., Tsuneta, S., \& Ogawara, Y. 1994, Nature, 371,495

McKenzie, D. E. 2000, Sol. Phys., 195, 381

McKenzie, D. E., \& Hudson, H. S. 2001, Earth, Planets, and Space, 53, 577

Milligan, R. O., McAteer, R. T. J., Dennis, B. R., \& Young, C. A. 2010, ApJ, 713, 1292

Narukage, N., \& Shibata, K. 2006, ApJ, 637, 1122

Newkirk, Jr., G. 1961, ApJ, 133, 983

Ning, Z., Wu, H., Xu, F., \& Meng, X. 2007, Sol. Phys., 241, 77

Nishida, K., Shimizu, M., Shiota, D., et al. 2009, ApJ, 690, 748

Nishizuka, N., Takasaki, H., Asai, A., \& Shibata, K. 2010, ApJ, 711, 1062

Ohyama, M., \& Shibata, K. 1998, ApJ, 499, 934

Oka, M., Phan, T.-D., Krucker, S., Fujimoto, M., \& Shinohara, I. 2010, ApJ, 714, 915

Parker, E. N. 1957, J. Geophys. Res., 62, 509

Parker, E. N. 1963, ApJS, 8, 177

Patsourakos, S., Vourlidas, A., \& Stenborg, G. 2013, ApJ, 764, 125

Petschek, H. E. 1964, NASA SP, 50, 425

Pohjolainen, S., van Driel-Gesztelyi, L., Culhane, J. L., Manoharan, P. K., \& Elliott, H. A. 2007, Sol. Phys., 244, 167

Pohjolainen, S., Pomoell, J., \& Vainio, R. 2008, A\&A, 490, 357

Priest, E., \& Forbes, T. 2000, Magnetic Reconnection (New York: Cambridge University Press)

Reeves, K. K., \& Golub, L. 2011, ApJ, 727, L52

Riley, P., Lionello, R., Mikić, Z., et al. 2007, ApJ, 655, 591

Samtaney, R., Loureiro, N. F., Uzdensky, D. A., Schekochihin, A. A., \& Cowley, S. C. 2009, Phys. Rev. Lett., 103, 105004

Savage, S. L., \& McKenzie, D. E. 2011, ApJ, 730, 98

Savage, S. L., McKenzie, D. E., Reeves, K. K., Forbes, T. G., \& Longcope, D. W. 2010, ApJ, 722, 329

Savage, S. L., Holman, G., Reeves, K. K., et al. 2012, ApJ, 754, 13

Scherrer, P. H., Schou, J., Bush, R. I., et al. 2012, Sol. Phys., 275, 207

Schou, J., Scherrer, P. H., Bush, R. I., et al. 2012, Sol. Phys., 275, 229

Shen, C., Lin, J., \& Murphy, N. A. 2011, ApJ, 737, 14

Shibata, K., \& Magara, T. 2011, Liv. Rev. Sol. Phys., 8, 6

Shibata, K., \& Tanuma, S. 2001, Earth, Planets, and Space, 53, 473

Shibata, K., Ishido, Y., Acton, L. W., et al. 1992, PASJ, 44, L173

Shibata, K., Masuda, S., Shimojo, M., et al. 1995, ApJ, 451, L83

Shimojo, M., Hashimoto, S., Shibata, K., et al. 1996, PASJ, 48, 123

Straka, R. M. 1977, in BAAS, 9, 245

Sturrock, P. A. 1966, Nature, 211, 695

Sui, L., \& Holman, G. D. 2003, ApJ, 596, L251

Sweet, P. A. 1958, in Electromagnetic Phenomena in Cosmical Physics, ed. B. Lehnert, IAU Symp., 6, 123

Takasao, S., Asai, A., Isobe, H., \& Shibata, K. 2012, ApJ, 745, L6

Török, T., Chandra, R., Pariat, E., et al. 2011, ApJ, 728, 65

Tsuneta, S., Hara, H., Shimizu, T., et al. 1992, PASJ, 44, L63

Ugai, M., \& Tsuda, T. 1977, J. Plas. Phys., 17, 337

Wang, R., Tan, B., Tan, C., \& Yan, Y. 2012, Sol. Phys., 278, 411

Yokoyama, T., \& Shibata, K. 1994, ApJ, 436, L197

Yokoyama, T., \& Shibata, K. 1997, ApJ, 474, L61

Yokoyama, T., \& Shibata, K. 1998, ApJ, 494, L113

Yokoyama, T., Akita, K., Morimoto, T., Inoue, K., \& Newmark, J. 2001, ApJ, 546, L69

Zhang, J., Cheng, X., \& Ding, M.-D. 2012, Nat. Comm., 3 